\title{
Microgrooving of Germanium Wafers Using Laser and Hybrid Laser-Waterjet Technologies
}

\author{
Hao Zhu' ${ }^{1, a}$, Jun Wang ${ }^{1, b,{ }^{*}}$, Weiyi $\mathrm{Li}^{1, \mathrm{c}}$, Huaizhong $\mathrm{Li}^{2, \mathrm{~d}}$ \\ ${ }^{1}$ School of Mechanical and Manufacturing Engineering, University of New South Wales, Australia \\ ${ }^{2}$ Griffith School of Engineering, Griffith University (Gold Coast Campus), Parklands Drive, Southport \\ Queensland 4214, Australia \\ ahao.zhu@student.unsw.edu.au, bjun.wang@unsw.edu.au, cweiyi.li@unsw.edu.au, \\ dlihuaizhong@gmail.com
}

Keywords: Germanium; Nanosecond laser; Hybrid laser-waterjet; Microgrooving; HAZ.

\begin{abstract}
Lasers have the potential for the micromachining of germanium (Ge). However, the thermal damages associated with the laser machining process need to be properly controlled. To minimize the thermal damages, a hybrid laser-waterjet ablation technology has recently been developed for micromachining. This paper presents an experimental study to assess the machining performances in microgrooving of Ge by using a nanosecond laser and by using the hybrid laser-waterjet technology. The effects of laser pulse energy, pulse overlap and focal plane position on the groove geometry and heat affected zone (HAZ) size are analyzed and discussed. It is shown that the hybrid laser-waterjet technology can give rise to narrow and deep microgrooves with minimum HAZ.
\end{abstract}

\section{Introduction}

Germanium (Ge) as a semiconductor material is becoming increasingly important to various applications. Comparing with silicon, it has the advantages of substantially higher electron and hole mobility which indicates potential for higher-speed devices. It has been used intensively in the fields of fiber-optic systems, infrared optics, electronics and solar electric applications [1-3]. However, Ge is very brittle and easy to get broken, which causes its processing a challenging task [4]. Ultrasonic elliptical vibration cutting [2] and diamond microgrooving [5] have been used to machine Ge in its ductile regime to achieve good quality with reduced fractures. However, these cutting methods are limited by the very small critical depth of cut, which is less than $2 \mu \mathrm{m}$ for Ge. In terms of non-mechanical machining, chemical etching of Ge is much more difficult and inefficient than that for Si as the former requires a high etching anisotropy to develop a V-groove, and special mask and specific acidic etchant must be prepared $[1,6]$. Wire electro-discharge machining (WEDM) showed good performance in Ge slicing [4], and thin wire was used to reduce wastage, but the low slicing rate is still a concern.

By contrast, laser machining is associated with acceptable processing rate and is commonly used in industry. However, as a thermal process through melt expulsion and vaporization of unwanted material, laser irradiation causes material properties in the surrounding area to change through heat effect and melt resolidification. As a result, the process introduces undesirable thermal damages manifested as HAZ, which reduces the functionality and reliability of the final products. Nanosecond pulsed lasers have been widely used in the micromachining of Si [7] and other brittle materials, and has a potential for effective Ge micromachining. However, the reported studies on micromachining of Ge using lasers are far from being comprehensive [8], and most studies focused on the damage mechanism of Ge instead of micromachining [9], although it has been reported that micro-deep cavities occur in the cut bottom, and rough surface morphology with cavities is formed, including wavelike features, droplets and cavities in nanosecond laser machining of Ge [10].

The hybrid laser-waterjet processing [11] provides a near damage-free micromachining technology by combining a laser for heating and softening the material and a waterjet for removing the laser-softened material. In micromachining $\mathrm{Si}$, it has been shown that the technology can remove the material in its soft solid status several hundred degrees below its melting temperature. The thermal damage as assessed by amorphous layer thickness is extremely small [12]. This paper 
presents a recent research to extend its application to Ge that has a lower melting point than Si. Experiments on micromachining of Ge by using a nanosecond laser in the laser dry cutting mode (or laser dry cutting) and using the hybrid laser-waterjet processing are carried out. The machining performance in terms of groove geometry and HAZ is evaluated. A comparison between the two processing methods is made to assess the advantages of the hybrid technology.

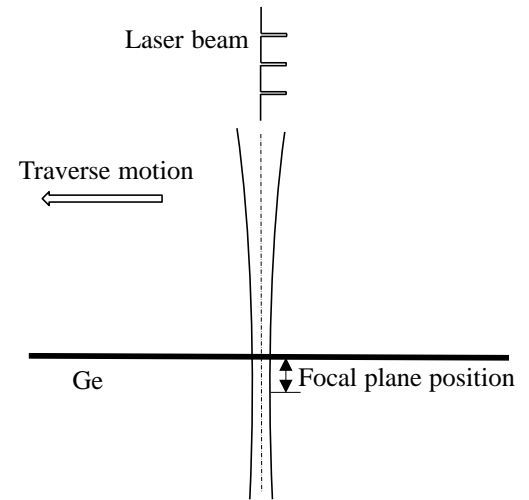

(a)

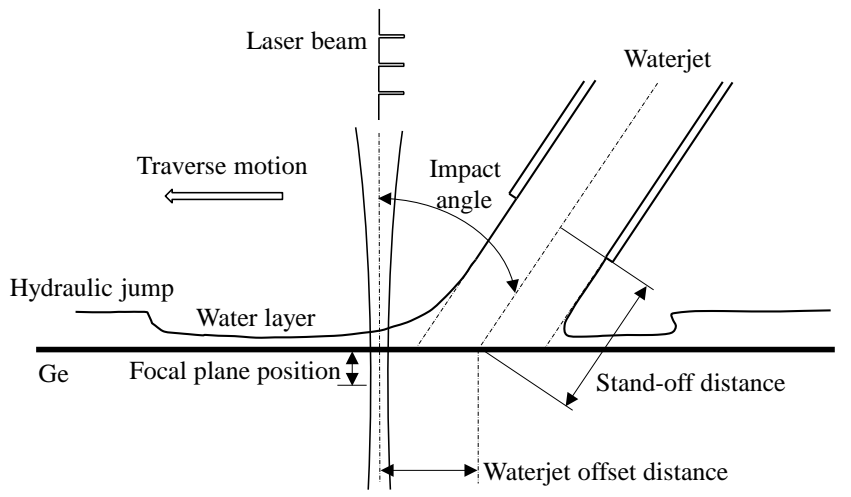

(b)

Fig. 1. Schematics of machining by (a) laser dry cutting and (b) hybrid laser-waterjet technology.

\section{Experimental methodology}

In this study, a $<100>$ oriented single crystalline Ge wafer with the thickness of $460 \mu \mathrm{m}$ was machined using a nanosecond laser in a dry cutting mode and the hybrid laser-waterjet cutting mode, respectively. The experimental setups are shown in Fig. 1. The laser machine used for both cutting modes was a 42 ns pulsed fiber laser with the Gaussian beam at $1080 \mathrm{~nm}$ wavelength and a random polarization. The focal diameter was adjusted to be $17.2 \mu \mathrm{m}$. The vertical position of the laser head can be manually adjusted via a Newport precision stage with a $1 \mu \mathrm{m}$ resolution. The focal plane position can be positive, zero and negative, where "positive" or "negative" corresponds to the focal plane being "above" or "below" the work surface, respectively. The waterjet offset distance was the distance between the central points of the laser beam and the waterjet on the specimen surface. The pulse overlap $(P O)$ represents the percentage of overlap between two consecutive pulses on the specimen surface, and was used as a non-dimensional term to consider the effect of laser pulse frequency $(f)$ and traverse speed $\left(v_{t}\right)$, i.e.

$$
P O=\left[1-\frac{v_{t}}{d_{b} f}\right] 100 \%,
$$

where $d_{b}$ is the laser beam diameter at the workpiece surface, which can be derived from laser wavelength $(\lambda)$, focal plane position ( $f p p)$ and focused laser beam diameter $\left(d_{f}\right)$ as.

$$
d_{b}=d_{f} \sqrt{1+\left(\frac{4 \lambda f p p}{\pi d_{f}^{2}}\right)^{2}} .
$$

The parameters used in this study are given in Table 1.

Table 1 Process parameters and the values used in the experiments

\begin{tabular}{lcccc}
\hline Focal plane position $(f p p)[\mathrm{mm}]$ & 0 & -0.2 & -0.4 & -0.6 \\
Laser pulse energy $(E p)[\mathrm{mJ}]$ & 0.3 & 0.43 & 0.56 & 0.7 \\
Laser pulse overlap $(P O)[\%]$ & 98.7 & 99 & 99.3 & 99.6 \\
Waterjet impact angle $\left(\theta_{j}\right)[\mathrm{deg}]$ & 45 & & & \\
Water pressure $\left(P_{w j}\right)[\mathrm{MPa}]$ & 6 & & & \\
\hline
\end{tabular}




$$
\text { Waterjet offset distance }\left(x_{w l}\right)[\mathrm{mm}] \quad 0.44
$$

A full-factorial design for the processing parameters in Table 1 resulted in 64 sets of combinations of the parameters for using each of the two machining technologies, respectively. The groove width, depth, HAZ size and surface morphology were measured and observed under a 3D laser microscope (Keyence VK-X200).

\section{Results and Discussion}

In the laser dry machining mode, as no assistant gas was used, the removal of material was achieved by the thermal process only. It was found that grooves could not be properly produced in many cases of laser drying cutting. Instead, humps were formed in the laser path on the Ge surface as a result of melt resolidification. For this analysis, the groove width, groove depth and HAZ size are used, as shown in Fig. 2 where the HAZ is defined as the distance from the edge of the pile-up (or hump) to where color transition on the Ge surface can be clearly seen (where most spatter spots are in fact included). A qualitative study of cutting results, as well as a quantitative comparison on HAZ size between the two processes is also made. They are discussed below.

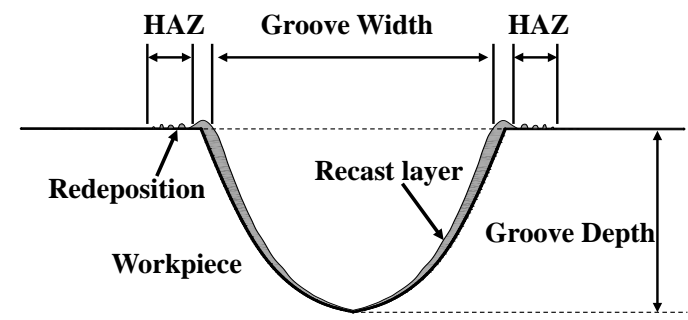

Fig. 2. Schematic of groove geometry and HAZ used in the analysis.

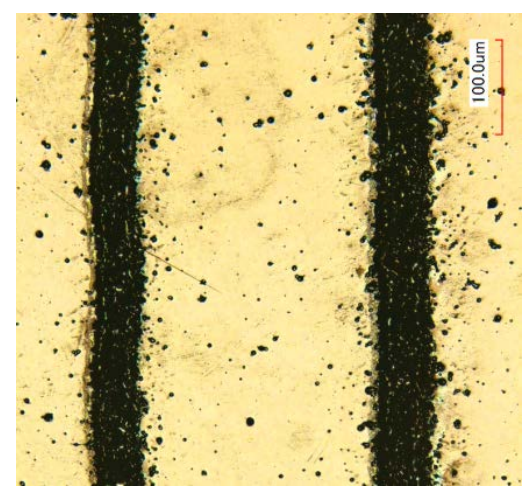

(a)

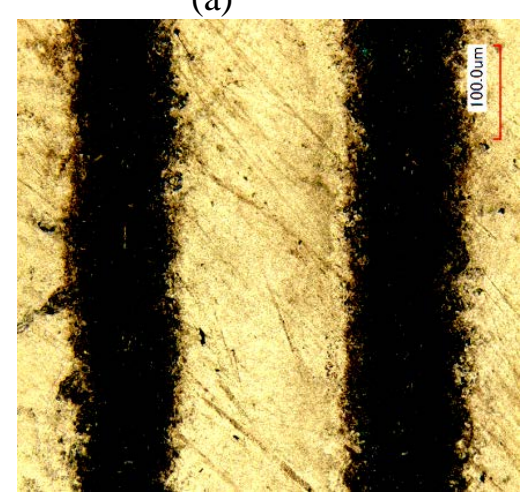

(c)

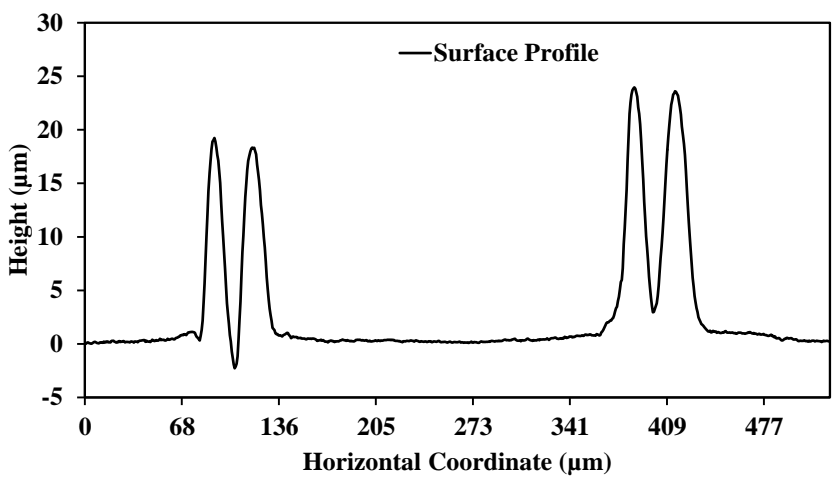

(b)

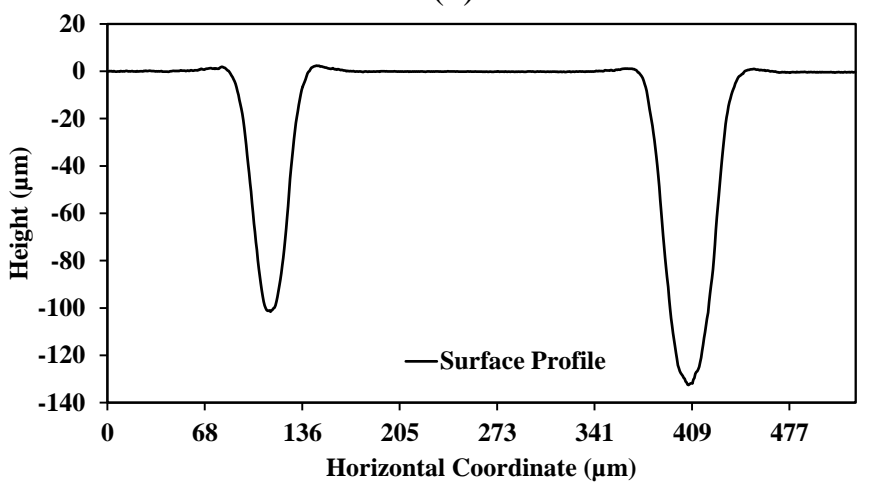

(d)

Fig. 3. Machined profiles on Ge ( $f p p=-0.2 \mathrm{~mm}, P O=99 \%, E p=0.3 \mathrm{~mJ}$ for the left cut and $E p=0.43$ $\mathrm{mJ}$ for the right cut; $\theta_{j}=45^{\circ}, P_{w j}=6 \mathrm{MPa}$, and $x_{w l}=0.44 \mathrm{~mm}$ for (c) and (d)): (a) top view from dry cutting; (b) sectional profile from dry cutting; (c) top view from hybrid cutting; and (d) sectional profile from hybrid cutting. 
The Groove Quality and Surface Morphology. The results from the laser dry cutting process are shown in Figs. 3(a) and (b). In most cases, grooves could not be produced under these dry cutting conditions. Instead, a hump was formed (Fig. 3(b)) and spatter points or deposited materials can be found in the surrounding area (Fig. 3(a)). This appears to be different from the grooving of Si under similar condition [11], since the different material properties have important effect on the performance of laser dry machining [13]. Generally in the pulse duration, the material is heated and expands firstly. It is then melted when the temperature reaches its melting point and vaporizes at its boiling point. The recoil pressure and thermo-capillary force [14] formed in the process, which depend on both the material properties and laser condition [15], supply the driving force for liquid ejection, thus the material is removed both in vapor and liquid forms. Besides the difference in thermal properties, Ge is roughly twice as dense as $\mathrm{Si}$ in both solid and liquid states, thus it is harder to eject molten Ge than molten Si under the same conditions. When the molten Ge cannot be removed in time, the mentioned driving force may act to reshape the molten material in the resolidification process, so that large pile-up may be formed, including humps.

By contrast, the possible inefficiency of molten material ejection does not exist in the hybrid cutting process, as the material removal mechanism of the latter process is due to the waterjet impingement, and the softened material is possible to be expelled in solid state [12]. Figs. 3(c) and (d) show the top views and sectional profiles of two grooves produced by the hybrid laser-waterjet process. It can be seen that this technology could indeed produce good quality grooves, and the work surface remains clean, although there is a small HAZ. Pile-ups, which are usually seen in the edges of cuts by the laser dry machining, are significantly reduced to a negligible level. The top groove width and groove depth can be reasonably controlled by selecting the appropriate process parameters and for almost all the grooves machined, the top groove widths are within $100 \mu \mathrm{m}$, while the groove depth is up to $300 \mu \mathrm{m}$. Under the impingement of a waterjet, the amount of material removal is determined by the temperature profile on the workpiece and the distribution of the waterjet pressure at the impact site. The factors affecting the heat source, impinging force and energy loss can affect the results in the hybrid laser-waterjet cutting process. The effects of laser pulse energy, pulse overlap and focal plane position on groove geometry are discussed below.

(a)

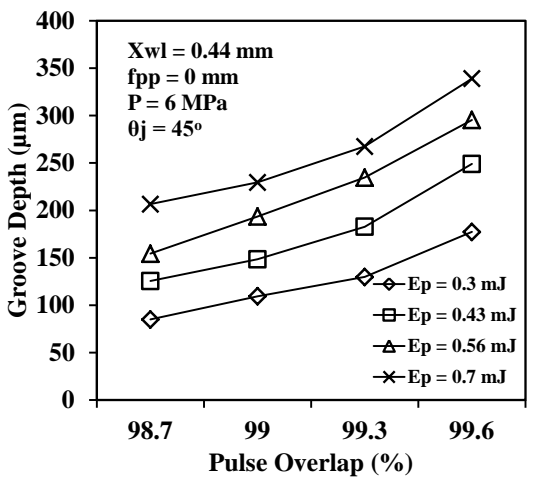

(c)

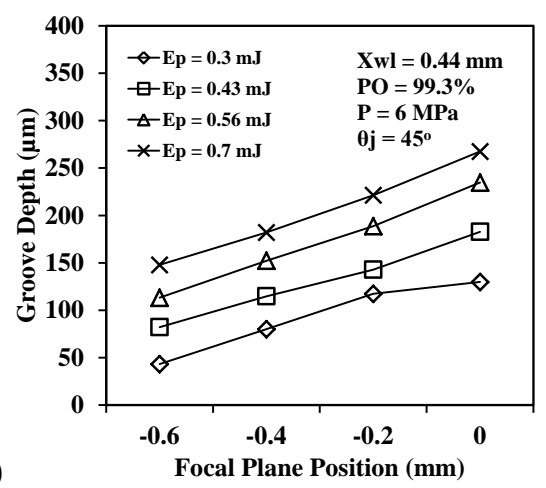

(b)
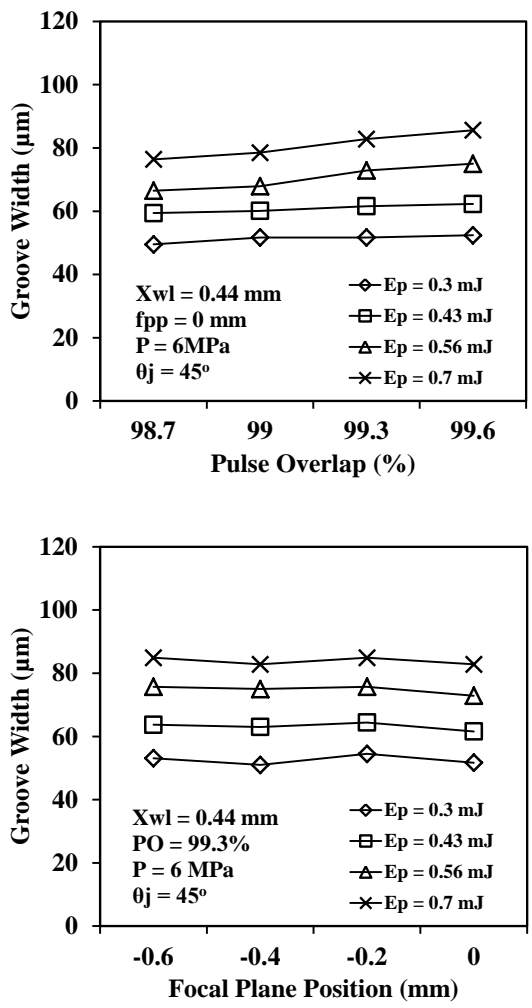

Fig. 4. Effect of process parameters on the groove geometry in hybrid laser-waterjet machining. 
Effect of the Process Parameters on the Groove Geometry. The effects of laser pulse energy, pulse overlap and focal plane position on groove geometries yielded by hybrid cutting process are shown in Fig. 4 . It is found that an increase in laser pulse energy $\left(E_{p}\right)$ consistently increases both the groove depth and width significantly, as a higher laser energy input causes an increased laser heated region and hence an enlarged groove. By contrast, the pulse overlap and focal plane position have a significant effect on the groove depth (Figs. 4(a) and (c)), but to a much lesser extent on the groove width (Figs. 4(b) and (d)). The increase of groove depth with pulse overlap was due to a higher pulse overlap that brings more input energy to a given area on the workpiece. The fact that the groove depth increases significantly when the focal plane position changed from -0.6 to $0 \mathrm{~mm}$ may be attributed to the reduced laser beam at the work surface that increases the laser intensity, but decreases the irradiated area. The small variation of groove width with the change in pulse overlap and focal plane position may be due to the small change in the effective beam diameter within which the material was softened enough for the removal by the waterjet, as these two parameters were changed. Further, the significant cooling effect of the waterjet helped heat loss on the work surface through forced convection [12]; thus reducing the heat conducted in groove width direction.

(a)

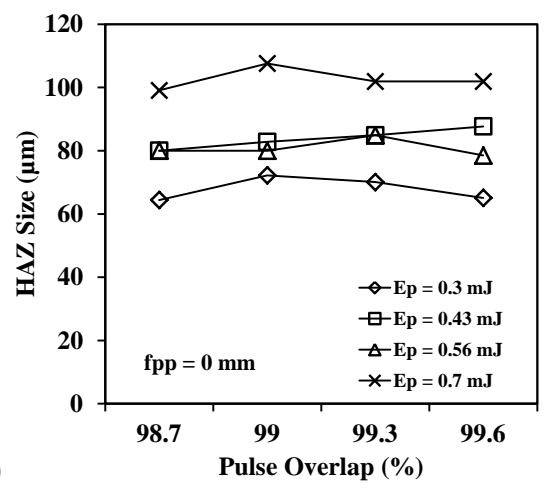

(c)

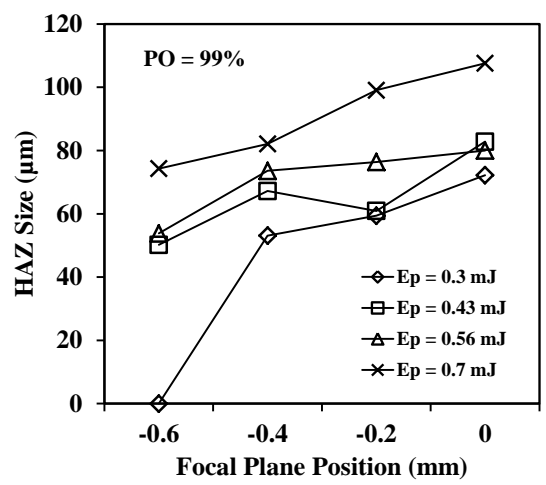

(b)

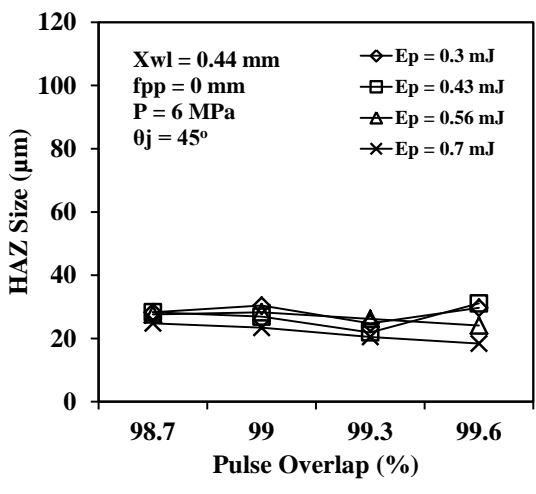

(d)

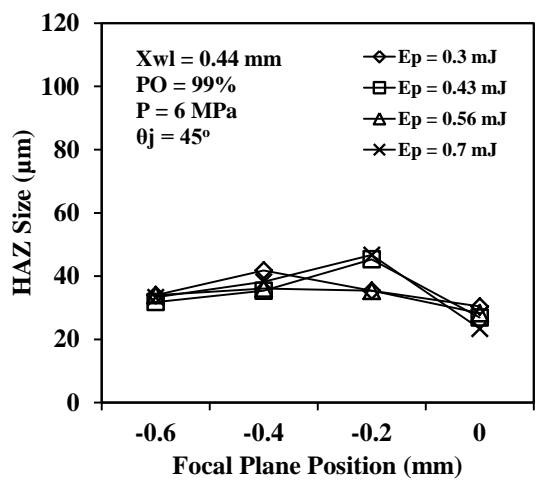

Fig. 5. Effect of process parameters on HAZ: (a) and (c) are for dry cutting, and (b) and (d) are for hybrid cutting.

Effect of the Process Parameters on the HAZ Size. It is apparent from Fig. 5 that the HAZ size from the hybrid process is significantly small compared to that from the dry cutting process due to both the forced convection and immediate removal of the hot and softened material by the waterjet. Further, the water layer formed on the work surface and the waterjet impact effect prevented spatter material from depositing on the surrounding area. It is interesting to note that while the change of pulse energy $\left(E_{p}\right)$ has a significant effect on the HAZ size in laser dry cutting, this effect is negligible in the hybrid process, again because of the waterjet cooling effect that suppressed the amount of heat conducted along the work surface and limited the HAZ size to somewhere very close to the groove. In both processes, the change of pulse overlap from $98.7 \%$ to $99.6 \%$ has a negligible effect on the HAZ size.

It is worth noting that a decrease in the focal plane position decreases the HAZ size in the laser dry cutting process, while its effect in the hybrid process is very marginal. It is found from the laser dry 
cutting process that the spatter phenomenon becomes bigger when the focal plane position increases from negative to zero; therefore, the HAZ that contains the spatter spots area as defined in this study becomes larger. By contrast, these spatter spots cannot deposit on surface in the hybrid process, thus the change of focal plane position has a negligible effect on the HAZ size.

\section{Conclusions}

An experimental study has been carried out to study the grooving process on Ge wafers by using a hybrid laser-waterjet cutting technology. For a comparison purpose, grooving using a nanosecond laser under the dry cutting mode was also conducted. It has been found that using laser dry cutting, grooves could not be produced in most conditions considered, while good quality V-shaped grooves have been made easily using the hybrid cutting technology. The HAZ size from the hybrid cutting process is significantly smaller than that from the laser dry cutting process. The effect of laser pulse energy, focal plane position and pulse overlap on groove geometry and HAZ size have been discussed. For the hybrid cutting process, laser pulse energy has a significant effect on both the groove width and depth. While the focal plane position and pulse overlap have a remarkable effect on groove depth, their effect on the groove width is limited. More studies to characterize the hybrid laser-waterjet micromachining process for Ge wafers are being carried out and the results will be reported shortly.

\section{References}

[1] R. Leancu, N. Moldovan, L. Csepregi and W. Lang, Sensors and Actuators A: Physical. 46(1-3) (1995), pp. 35-37.

[2] N. Suzuki, Z. Yan, R. Hino, E. Shamoto, and Y. Hirahara, In Conference Record of the 2006 International Symposium on Micro-NanoMechatronics and Human Science, NGO, (2006).

[3] E.E. Haller, Material Science in Semiconductor Processing. 9(4-5) (2006), pp. 408-422.

[4] D. Rakwal, E. Bamberg, Journal of Materials Processing Technology. 209(8) (2009), pp. 3740-3751.

[5] J. Yan, K. Maekawa, J. Tamaki and T. Kuriyagawa, Journal of Micromechanics and Microengineering. 15(10) (2005), pp. 1925-1931.

[6] W. Lang, R. Leancu, U. Schaber, G. Wiedamann and H.U. Kaufl, Journal of Micromechanics and Microengineering. 6(1) (1996), pp. 46-48.

[7] L.M. Wee, E.Y.K. Ng, A.H. Prathama and H. Zheng, Optics \& Laser Technology. 43(1) (2011), pp. 62-71.

[8] S. Manicham, J. Wang and C.Z. Huang, Journal of Engineering Manufacture. 227(11) (2013), pp. 1714-1723.

[9] K. Diener, L. Gernandt, J.-P. Moeglin and P. Ambs, Optics and Lasers in Engineering. 43(11) (2005), pp. 1179-1192.

[10] V. Craciun, D. Craciun, M.C. Bunescu, C. Boulmer-Leborgne and J. Hermann, Physical Review B. 58(11) (1998), pp. 6787-6790.

[11] V. Tangwarodomnukun, J. Wang, C.Z. Huang and H.T. Zhu, International Journal of Machine Tools \& Manufacture. 56 (2012), pp. 39-49.

[12] V. Tangwarodomnukun, J. Wang, C.Z. Huang and H.T. Zhu, International Journal of Machine Tools \& Manufacture. 79 (2014), pp. 1-16.

[13] F.P. Gagliano and U.C. Paek, Applied Optics. 13(2) (1974), pp. 274-279.

[14] Q. Wu, J. Wang and C.Z. Huang, Proc IMechE Part B: Journal of Engineering Manufacture, 228(6) (2014), pp. 903-917.

[15] V. Semak and A. Matsunawa, Journal of Physics D: Applied Physics. 30 (1997), pp. 2541-2552. 